\title{
STABILITY ANALYSIS OF HIGH-ORDER HOPFIELD-TYPE NEURAL NETWORKS BASED ON A NEW IMPULSIVE DIFFERENTIAL INEQUALITY
}

\author{
YAng LIU ${ }^{*}$, RongJiang YANG *, Jianquan LU ${ }^{* *}$, Bo WU ${ }^{*, * * *}$, XiUshan CAI ${ }^{*}$ \\ * College of Mathematics, Physics and Information Engineering \\ Zhejiang Normal University, Jinhua 321004, China \\ e-mail: liuyang4740@gmail.com \\ ** Department of Mathematics \\ Southeast University, Nanjing 210096, China \\ e-mail: jqluma@seu.edu.cn \\ *** Academic Affairs Division \\ Zhejiang Normal University, Jinhua 321004, China \\ e-mail: wubo_502@163.com
}

\begin{abstract}
This paper is devoted to studying the globally exponential stability of impulsive high-order Hopfield-type neural networks with time-varying delays. In the process of impulsive effect, nonlinear and delayed factors are simultaneously considered. A new impulsive differential inequality is derived based on the Lyapunov-Razumikhin method and some novel stability criteria are then given. These conditions, ensuring the global exponential stability, are simpler and less conservative than some of the previous results. Finally, two numerical examples are given to illustrate the advantages of the obtained results.
\end{abstract}

Keywords: impulsive differential inequality, globally exponential stability, high-order Hopfield-type neural network.

\section{Introduction}

Hopfield neural networks have been extensively studied and developed in recent years, and there has been considerable focus in the literature on Hopfield neural networks (Cao and Xiao, 2007; Wallis, 2005). It is known that high-order neural networks, including high-order Hopfield neural networks, have stronger approximation property, faster convergence rate, greater storage capacity, and higher fault tolerance than low-order neural networks, and hence they have been studied by many researchers (Ho et al., 2006; Liu and Wang, 2008; Liu et al., 2005; Ren and Cao, 2006; Xu et al., 2003; 2009).

Due to limited computational time and network environment, time delays are always inevitable (Tian et al., 2004; Zhang and Sun, 2010; Weng and Sun, 2009; Khadra et al., 2009; Liu et al., 2007; Wu et al., 2012b), especially in the interaction of neurons in biological and artificial neural networks (Cao and Xiao, 2007; Ho et al., 2006; Ren and Cao, 2006; Xu et al., 2003; 2009; Stamova and Ilarionov, 2010; Chua an Yang,
$1988 b ; 1988 a)$. The existence of delays is frequently a source of instability for neural networks. Fixed and time-varying delays have been considered in the literature concerning the stability of Hopfield-type neural networks (Civalleri et al., 1993; Gopalsamy and He, 1994; Lou and Cui, 2007; van den Driessche and Zou, 1998). If an equilibrium point of a Hopfield neural network is globally exponentially stable, then the domain of attraction of the equilibrium point is the whole space. This is of importance from both a theoretical and an application oriented point of view. On the other hand, there are many abruptly changing phenomena for the states of biological, economical, control, and telecommunication systems, which can be well described by impulsive ones. Systematic analysis of impulsive systems has been widely considered by Huang and Yang (2010), Yang and Chua (1997), Wu et al. (2011; 2012a), Lu et al. (2010; 2011; 2012), Liu et al. (2011), Raja et al. (2011) and Sakthivel et al. (2010a; 2010b; 2010c; 2011).

In the literature, various methods and techniques 
have been effectively utilized for stability analysis of neural networks, for example, the Linear Matrix Inequality (LMI) approach (Liu et al., 2005; Ren and Cao, 2006; Xu et al., 2003; 2011; Raja et al., 2011; Sakthivel et al., 2011; 2010c; 2010a; 2010b; Rong, 2005; Zhang et al., 2010; Zheng et al., 2011), the Lyapunov-Razumikhin method (Liu and Wang, 2008; Stamova and Ilarionov, 2010; Stamova et al., 2010; Ahmad and Stamova, 2008) the Lyapunov functional method (Xu et al., 2003; 2011; He et al., 2006), the use of properties of the M-matrix (Xu et al., 2009; Chen, 2001), and differential inequalities (Xu et al., 2009; Zhang et al., 2010; Li et al., 2008; Li, 2010). The importance of impulses with time delay lies in the fact that they can be used to model the impulsive controller subject to transmission or sampling delays (Khadra et al., 2009; Zhang et al., 2011). Hence, there are many studies on the stability of neural networks with delayed impulses (Ho et al., 2006; Liu et al., 2005). However, the results on the stability of neural networks of Ho et al. (2006), Liu and Wang (2008), Liu et al. (2005; 2007), Xu et al. (2009), Khadra et al. (2009), He et al. (2006), Li (2010) or Wang and Liu (2007) impose some restrictions on delays in terms of its relation to the impulse intervals, while the results of Ren and Cao (2006) as well as Zheng et al. (2011) require some conditions on the increasing of time-varying delays. Hence, the existing research on stability of neural networks with time-delay and impulses may still be insufficient.

Motivated by the above discussions, we employ a new Lyapunov-based impulsive differential inequality with delays, which is different from that of Ho et al. (2006), Liu et al. (2005), Xu et al. (2009), Li (2010), Yang and Xu (2005; 2007), Zhou and Wu (2009) or Yue et al. (1999) and extends the famous Halanay differential inequality. Even when nonlinear and delay effects are simultaneously considered in the process of impulses, our new impulsive differential inequality can be used to derive novel stability criteria which can remove some complex restrictions concerning the relations between impulse times and delays imposed by Ho et al. (2006), Liu and Wang (2008), Liu et al. (2005; 2007), Xu et al. (2009), Khadra et al. (2009), He et al. (2006), Li (2010) or Wang and Liu (2007). For example, the impulsive interval should be bigger than the upper bound of the delay function given by Ho et al. (2006), Liu and Wang (2008) or Liu et al. (2005). Furthermore, the proposed results do not require the decreasing of the Lyapunov functions during the continuous segment of the trajectory all the time, which is required by Stamova and Ilarionov (2010), Huang and Yang (2010), Stamova et al. (2010) or Ahmad and Stamova (2008). In addition, the requirement on the increasing of time-varying delays as in the works of Ren and Cao (2006) as well as Zheng et al. (2011) is not needed in the obtained results. Hence, the impulsive differential inequality presented in the paper can be applied to analyze the stability of neural networks to get less conservative sufficient conditions and used to investigate the stability of genetic regulatory networks, which will be studied in our future research.

The rest of this paper is organized as follows. In Section 2, we introduce some notations and definitions. Impulsive high-order Hopfield-type neural networks with time-varying delays are described. In Section 3, we obtain several exponential stability criteria for high-order Hopfield-type neural networks based on a new impulsive differential inequality. In Section 4 two examples are presented to illustrate the advantages of our results. Conclusions are given in the last section.

\section{Preliminaries}

Consider the impulsive high-order Hopfield-type neural network with time-varying delays described by the following impulsive differential equation:

$$
\left\{\begin{aligned}
C_{i} u_{i}^{\prime}(t)= & -\frac{u_{i}(t)}{R_{i}}+\sum_{j=1}^{n} P_{i j} g_{j}\left(u_{j}\left(t-\tau_{j}(t)\right)\right) \\
& +\sum_{j=1}^{n} \sum_{l=1}^{n} P_{i j l} g_{j}\left(u_{j}\left(t-\tau_{j}(t)\right)\right) \\
& \times g_{l}\left(u_{l}\left(t-\tau_{l}(t)\right)\right)+I_{i}, \quad t \in\left[t_{k-1}, t_{k}\right), \\
\Delta u_{i}(t)= & d_{i k} u_{i}\left(t^{-}\right)+\sum_{j=1}^{n} W_{i j}^{(k)} h_{j}\left(u_{j}\left(t^{-}-\tau_{j}(t)\right)\right) \\
& +\sum_{j=1}^{n} \sum_{l=1}^{n} W_{i j l}^{(k)} h_{j}\left(u_{j}\left(t^{-}-\tau_{j}(t)\right)\right) \\
& \times h_{l}\left(u_{l}\left(t^{-}-\tau_{l}(t)\right)\right), \quad t=t_{k}, k \in \mathbb{N},
\end{aligned}\right.
$$

where $i \in J=\{1,2, \ldots, n\}, \Delta u_{i}\left(t_{k}\right)=u_{i}\left(t_{k}\right)-$ $u_{i}\left(t_{k}^{-}\right), u_{i}\left(t_{k}^{-}\right)=\lim _{t \rightarrow t_{k}^{-}} u_{i}(t), k \in \mathbb{N}$. The time sequence $\left\{t_{k}\right\}$ satisfies $0 \leq t_{0}<t_{1}<t_{2}<\cdots$, with $\lim _{k \rightarrow \infty} t_{k}=\infty ; C_{i}>0, R_{i}>0$ and $I_{i}$ are the capacitance, the resistance and the external input of the $i$-th neuron, respectively. The matrices $P_{i j}, W_{i j}^{(k)}$ and $P_{i j l}, W_{i j l}^{(k)}$ are the first and second-order synaptic weights of the neural network, and $\tau_{i}(t), i \in J$ is the transmission delay of the $i$-th neuron such that $0 \leq \tau_{i}(t) \leq \tau$, where $\tau$ is a constant.

For simplicity, denote by $\mathbb{R}^{+}$the set of nonnegative real numbers and by $\mathbb{R}^{n}$ the $n$-dimensional real space. Use $\lambda_{\min }(P)$ and $\lambda_{\max }(P)$ to denote, respectively, the smallest and the largest eigenvalues of a square matrix $P$. $I$ denotes the identity matrix of degree $n$. The norm $\|\cdot\|$ is either any vector norm or the induced matrix norm. Furthermore, $\operatorname{diag}\left(a_{1}, a_{2}, \ldots, a_{n}\right)$ denotes the diagonal matrix with elements in diagonal equal to $a_{1}, a_{2}, \ldots, a_{n}$.

Throughout this paper, we assume that the neuron activation functions $g_{i}(u)$ and $h_{i}(u)$, satisfy the following 
conditions with constants $M_{i}, N_{i}, K_{i}, L_{i}, i \in J$ :

$$
\begin{aligned}
\left|g_{i}\left(u_{i}\right)\right| & \leq M_{i} \quad\left|h_{i}\left(u_{i}\right)\right| \leq N_{i}, \quad \forall u_{i} \in \mathbb{R} ; \\
0 & \leq \frac{g_{i}\left(u_{i}\right)-g_{i}\left(v_{i}\right)}{u_{i}-v_{i}} \leq K_{i}, \\
0 & \leq \frac{h_{i}\left(u_{i}\right)-h_{i}\left(v_{i}\right)}{u_{i}-v_{i}} \leq L_{i},
\end{aligned}
$$

for all $u_{i} \neq v_{i}, u_{i}, v_{i} \in \mathbb{R}, i \in J$.

It should be noticed that above assumptions are commonly used by Ho et al. (2006), Liu and Wang (2008), Liu et al. (2005), Xu et al. (2011) and Zheng et al. (2011). Define

$$
\begin{aligned}
M & =\left[M_{1}, M_{2}, \ldots, M_{n}\right]^{T}, \\
N & =\left[N_{1}, N_{2}, \ldots, N_{n}\right]^{T}, \\
K & =\operatorname{diag}\left(K_{1}, K_{2}, \ldots, K_{n}\right), \\
L & =\operatorname{diag}\left(L_{1}, L_{2}, \ldots, L_{n}\right) .
\end{aligned}
$$

The initial condition for the system (1) is given by $u_{i}(s)=\psi_{i}(s), s \in\left[t_{0}-\tau, t_{0}\right]$, where $\psi_{i}:\left[t_{0}-\tau, t_{0}\right] \rightarrow$ $\mathbb{R}, i=1,2, \ldots, n$, is a piecewise continuous function. From the work of $\mathrm{Xu}$ et al. (2009), the system (1) has an equilibrium point under the condition (2). Let $u^{*}=$ $\left[u_{1}^{*}, u_{2}^{*}, \cdots, u_{n}^{*}\right]^{T}$ be an equilibrium point of system (1). For $i \in J$ and $k \in \mathbb{N}$, let

$$
\begin{aligned}
& x_{i}(t)=u_{i}(t)-u_{i}^{*}, \\
& d_{i k} u_{i}^{*}+\sum_{j=1}^{n} W_{i j}^{(k)} h_{j}\left(u_{j}^{*}\right) \\
& \quad+\sum_{j=1}^{n} \sum_{l=1}^{n} W_{i j l}^{(k)} h_{j}\left(u_{j}^{*}\right) h_{l}\left(u_{l}^{*}\right)=0, \\
& f_{i}\left(x_{i}\left(t-\tau_{i}(t)\right)\right)=g_{i}\left(u_{i}\left(t-\tau_{i}(t)\right)\right)-g_{i}\left(u_{i}^{*}\right), \\
& \varphi_{i}\left(x_{i}\left(t-\tau_{i}(t)\right)\right)=h_{i}\left(u_{i}\left(t-\tau_{i}(t)\right)\right)-h_{i}\left(u_{i}^{*}\right) .
\end{aligned}
$$

Then, for each $i \in J$,

$$
\begin{array}{ll}
\left|f_{i}(z)\right| \leq K_{i}|z| \text { and } z f_{i}(z) \geq 0, & \forall z \in \mathbb{R}, \\
\left|\varphi_{i}(z)\right| \leq L_{i}|z| \text { and } z \varphi_{i}(z) \geq 0, & \forall z \in \mathbb{R} .
\end{array}
$$

The system (1) can be rewritten as follows:

$$
\left\{\begin{aligned}
C_{i} x_{i}^{\prime}(t)= & -\frac{x_{i}(t)}{R_{i}}+\sum_{j=1}^{n}\left(P_{i j}+\sum_{l=1}^{n}\left(P_{i j l}+P_{i l j}\right) \zeta_{l}\right) \\
& \times f_{j}\left(x_{j}\left(t-\tau_{j}(t)\right)\right), \quad t \in\left[t_{k-1}, t_{k}\right), \\
\Delta x_{i}\left(t_{k}\right)= & d_{i k} x_{i}\left(t_{k}^{-}\right)+\sum_{j=1}^{n}\left(W_{i j}^{(k)}\right. \\
& \left.+\sum_{l=1}^{n}\left(W_{i j l}^{(k)}+W_{i l j}^{(k)}\right) \xi_{l}\right) \\
& \times \varphi_{j}\left(x_{j}\left(t_{k}^{-}-\tau_{j}\left(t_{k}\right)\right)\right), \quad k \in \mathbb{N},
\end{aligned}\right.
$$

where $i \in J, \zeta_{l}$ is between $g_{l}\left(u_{i}\left(t-\tau_{l}(t)\right)\right)$ and $g_{l}\left(u_{l}^{*}\right)$, and $\xi_{l}$ is between $h_{l}\left(u_{l}\left(t_{k}^{-}-\tau_{l}\left(t_{k}\right)\right)\right)$ and $h_{l}\left(u_{l}^{*}\right)$. Define

$$
\begin{aligned}
C & =\operatorname{diag}\left(C_{1}, C_{2}, \ldots, C_{n}\right), \\
R & =\operatorname{diag}\left(R_{1}, R_{2}, \ldots, R_{n}\right), \\
D_{k} & =\operatorname{diag}\left(d_{1 k}, d_{2 k}, \ldots, d_{n k}\right), \\
W^{(k)} & =\left(W_{i j}^{(k)}\right)_{n \times n}, \\
W_{i}^{(k)} & =\left(W_{i j l}^{(k)}\right)_{n \times n}, \quad P=\left(P_{i j}\right)_{n \times n}, \\
P_{i}= & \left(P_{i j l}\right)_{n \times n}, \quad i \in J, \\
P_{H}= & {\left[P_{1}+P_{1}^{T}, \ldots, P_{n}+P_{n}^{T}\right]^{T}, } \\
\Xi^{(k)}= & {\left[W_{1}^{(k)}+\left[W_{1}^{(k)}\right]^{T}, \ldots,\right.} \\
& \left.W_{n}^{(k)}+\left[W_{n}^{(k)}\right]^{T}\right]^{T}, \\
\varphi\left(x\left(t^{-}-\tau(t)\right)\right)= & {\left[\varphi_{1}\left(x_{1}\left(t^{-}-\tau_{1}(t)\right)\right), \ldots,\right.} \\
& \left.\varphi_{n}\left(x_{n}\left(t^{-}-\tau_{n}(t)\right)\right)\right]^{T}, \\
f(x(t-\tau(t)))= & {\left[f_{1}\left(x_{1}\left(t-\tau_{1}(t)\right)\right), \ldots,\right.} \\
& \left.f_{n}\left(x_{n}\left(t-\tau_{n}(t)\right)\right)\right]^{T}, \\
\zeta= & {\left[\zeta_{1}, \ldots, \zeta_{n}\right]^{T}, } \\
\Gamma= & \operatorname{diag}(\zeta, \ldots, \zeta), \xi=\left[\xi_{1}, \ldots, \xi_{n}\right]^{T}, \\
\Lambda & =\operatorname{diag}(\xi, \ldots, \xi), \\
\Delta x= & {\left[\Delta x_{1}, \ldots, \Delta x_{n}\right]^{T}, } \\
x(t-\tau(t))= & {\left[x_{1}\left(t-\tau_{1}(t)\right), \ldots, x_{n}\left(t-\tau_{n}(t)\right)\right]^{T} . }
\end{aligned}
$$

Then the system (4) can be written in the following equivalent form:

$$
\left\{\begin{array}{rl}
x^{\prime}(t)=-C^{-1} R^{-1} & x(t)+C^{-1}\left(P+\Gamma^{T} P_{H}\right) \\
& \times f(x(t-\tau(t))), t \in\left[t_{k-1}, t_{k}\right), \\
\Delta x\left(t_{k}\right)=D_{k} x\left(t_{k}^{-}\right)+ & \left(W^{(k)}+\Lambda^{T} \Xi_{k}\right) \\
& \times \varphi\left(x\left(t_{k}^{-}-\tau\left(t_{k}\right)\right)\right), k \in \mathbb{N} .
\end{array}\right.
$$

The initial condition for the system (5) is given by $x(t)=$ $\phi(t), t \in\left[t_{0}-\tau, t_{0}\right]$, where

$$
\phi(t)=\left[\phi_{1}(t), \ldots, \phi_{n}(t)\right]^{T}, \quad t \in\left[t_{0}-\tau, t_{0}\right]
$$

and

$$
\phi_{i}(t)=\psi_{i}(t)-u_{i}^{*}, \quad t \in\left[t_{0}-\tau, t_{0}\right], i \in J .
$$

Now we introduce some definitions as follows.

Definition 1. A function $V: \mathbb{R}^{+} \times \mathbb{R}^{n} \rightarrow \mathbb{R}^{+}$is said to belong to class $V_{0}$ if

(i) $V$ is continuous in each of the sets $\left[t_{k-1}, t_{k}\right) \times \mathbb{R}^{n}$ and for each $x \in \mathbb{R}^{n}, t \in\left[t_{k-1}, t_{k}\right), k \in \mathbb{N}$, $\lim _{(t, y) \rightarrow\left(t_{k}^{-}, x\right)} V(t, y)=V\left(t_{k}^{-}, x\right)$ exists;

(ii) $V(t, x)$ is locally Lipschizian in all $x \in \mathbb{R}^{n}$, and for all $t \geq t_{0}, V(t, 0) \equiv 0$. 
Definition 2. Given a function $V: \mathbb{R}^{+} \times \mathbb{R}^{n} \rightarrow \mathbb{R}^{+}$, the upper right-hand derivative of $V \in V_{0}$ with respect to the system (1) is defined by

$$
\begin{aligned}
& D^{+} V(t, x(t)) \\
& =\limsup _{h \rightarrow 0^{+}} \frac{1}{h}[V(t+h, x(t+h))-V(t, x(t))] \\
& \text { for }(t, x) \in\left[t_{k-1}, t_{k}\right) \times \mathbb{R}^{n}, k \in \mathbb{N} \text {. }
\end{aligned}
$$

Definition 3. The trivial solution of the system (5) is said to be globally exponentially stable if there exist some constants $\alpha>0$ and $\tilde{M} \geq 1$ such that, for any initial data $x_{t_{0}}=\phi$,

$$
\left\|x\left(t, t_{0}, \phi\right)\right\| \leq \tilde{M}\|\phi\|_{\tau} e^{-\alpha\left(t-t_{0}\right)}, \quad t \geq t_{0},
$$

where

$$
\begin{gathered}
\left(t_{0}, \phi\right) \in \mathbb{R}^{+} \times P C\left([-\tau, 0], \mathbb{R}^{n}\right), \\
\|\phi\|_{\tau}=\sup _{s \in[-\tau, 0]}\|\phi(s)\| .
\end{gathered}
$$

Furthermore, $\alpha$ is called the convergence rate.

\section{Main results}

It is well known that a differential inequality (called the Halanay inequality (Halanay, 1966)) is one of the main tools for studying continuous differential systems and has been widely applied to stability analysis. However, these inequalities cannot be applied to impulsive differential systems directly. Furthermore, some previous results about differential inequalities cannot be used to deal with impulsive systems with time delay which appears both in the continuous and the impulsive part of the system. To overcome these difficulties, we give the following new impulsive differential inequality.

Lemma 1. Consider the following impulsive differential inequalities:

$$
\left\{\begin{aligned}
D^{+} v(t) & \leq \alpha v(t)+\beta \bar{v}(t), \quad\left[t_{k-1}, t_{k}\right) \\
v\left(t_{k}\right) & \leq a_{k} v\left(t_{k}^{-}\right)+b_{k} \bar{v}\left(t_{k}^{-}\right), \quad k \in \mathbb{N}
\end{aligned}\right.
$$

where $v(t) \geq 0$,

$$
\begin{gathered}
\bar{v}(t)=\sup _{t-\tau \leq s \leq t} v(s), \\
\bar{v}\left(t^{-}\right)=\sup _{t^{-}-\tau \leq s<t^{-}} v(s)
\end{gathered}
$$

and $v(t)$ is continuous except at each $t_{k}, k \in \mathbb{N}$. The sequence $\left\{t_{k}\right\}$ is assumed to satisfy $0 \leq t_{0}<t_{1}<\cdots<$ $t_{k}<\cdots$, and $\lim _{k \rightarrow \infty} t_{k}=\infty$.

Suppose that (i) there exists $\sigma>0$ and $\lambda>0$ such that

$$
\alpha+\left(\beta e^{\lambda \tau}\right) / d_{k-1} \leq \sigma-\lambda,
$$

where $d_{k} \geq a_{k}+b_{k} e^{\lambda \tau}, 0<d_{0} \leq 1, k \in \mathbb{N}$;

(ii) $\ln d_{k-1}<-(\sigma+\lambda)\left(t_{k}-t_{k-1}\right), k \in \mathbb{N}$.

Then there exists an $\tilde{M}>1$ such that $v(t) \leq$ $\tilde{M} \bar{v}\left(t_{0}\right) e^{-\lambda\left(t-t_{0}\right)}, t \in\left[t_{k-1}, t_{k}\right), k \in \mathbb{N}$.

Proof. Let $\gamma=\sup _{k \in \mathbb{N}}\left\{1 / d_{k-1}\right\}$. From (ii), we can get

$$
\ln \gamma+\lambda \tau-(\sigma+\lambda)\left(t_{k}-t_{k-1}\right)>0 .
$$

Hence, we can choose $\tilde{M} \geq 1$ such that

$$
\begin{aligned}
e^{(\sigma+\lambda)\left(t_{1}-t_{0}\right)} & \leq \tilde{M} \\
& \leq \gamma e^{\lambda \tau-(\sigma+\lambda)\left(t_{1}-t_{0}\right)} e^{(\sigma+\lambda)\left(t_{1}-t_{0}\right)} \\
& =\gamma e^{\lambda \tau} .
\end{aligned}
$$

From it follows (7) that

$$
\bar{v}\left(t_{0}\right)<\bar{v}\left(t_{0}\right) e^{\sigma\left(t_{1}-t_{0}\right)} \leq \tilde{M} \bar{v}\left(t_{0}\right) e^{-\lambda\left(t_{1}-t_{0}\right)} .
$$

We first show that

$$
v(t) \leq \tilde{M} \bar{v}\left(t_{0}\right) e^{-\lambda\left(t-t_{0}\right)}, \quad t \in\left[t_{0}, t_{1}\right) .
$$

To do this, we only need to prove

$$
v(t) \leq \tilde{M} \bar{v}\left(t_{0}\right) e^{-\lambda\left(t_{1}-t_{0}\right)}, \quad t \in\left[t_{0}, t_{1}\right) .
$$

If (9) is not true, from (8) there exists $\bar{t} \in\left(t_{0}, t_{1}\right)$ such that

$$
v(\bar{t})>\tilde{M} \bar{v}\left(t_{0}\right) e^{-\lambda\left(t_{1}-t_{0}\right)}>\bar{v}\left(t_{0}\right),
$$

which implies that there exists some $\hat{t} \in\left(t_{0}, \bar{t}\right)$ such that

$$
\begin{aligned}
v(\hat{t})=\tilde{M} \bar{v}\left(t_{0}\right) e^{-\lambda\left(t_{1}-t_{0}\right)}, & \\
& v(t) \leq v(\hat{t}), \quad t \in\left[t_{0}-\tau, \hat{t}\right],
\end{aligned}
$$

and there exists some $\tilde{t} \in\left(t_{0}, \hat{t}\right)$ such that

$$
v(\tilde{t})=\bar{v}\left(t_{0}\right), \quad v(\tilde{t}) \leq v(t) \leq v(\hat{t}), \quad t \in[\tilde{t}, \hat{t}] .
$$

Hence, by (7) and (10), for any $t \in[\tilde{t}, \hat{t}]$ and $s \in$ $[-\tau, 0]$, we have

$$
\begin{aligned}
v(t+s) & \leq \tilde{M} \bar{v}\left(t_{0}\right) e^{-\lambda\left(t_{1}-t_{0}\right)} \\
& \leq \gamma e^{\lambda \tau} \bar{v}\left(t_{0}\right) \\
& =\gamma e^{\lambda \tau} v(\tilde{t}) \\
& \leq \gamma e^{\lambda \tau} v(t), \quad s \in[-\tau, 0) .
\end{aligned}
$$

By the condition (i) and (6), we get

$$
\begin{aligned}
D^{+} v(t) & \leq \alpha v(t)+\beta \bar{v}(t) \\
& \leq \alpha v(t)+\beta \gamma e^{\lambda \tau} v(t) \\
& \leq(\sigma-\lambda) v(t), \quad t \in[\tilde{t}, \hat{t}] .
\end{aligned}
$$


Then, by (8),

$$
\begin{aligned}
v(\hat{t}) & \leq v(\tilde{t}) e^{(\sigma-\lambda)(\hat{t}-\tilde{t})}=\bar{v}\left(t_{0}\right) e^{(\sigma-\lambda)(\hat{t}-\tilde{t})} \\
& <\bar{v}\left(t_{0}\right) e^{\sigma\left(t_{1}-t_{0}\right)} \leq \tilde{M} \bar{v}\left(t_{0}\right) e^{-\lambda\left(t_{1}-t_{0}\right)}=v(\hat{t}),
\end{aligned}
$$

which is a contradiction. Hence, (9) holds.

Next, we shall show that

$$
v(t) \leq \tilde{M} \bar{v}\left(t_{0}\right) e^{-\lambda\left(t-t_{0}\right)}, \quad t \in\left[t_{k-1}, t_{k}\right), k \in \mathbb{N} .
$$

Assuming (11) holds for $k=1,2, \ldots, m$, we shall show that it holds for $k=m+1$, i.e.,

$$
v(t) \leq \tilde{M} \bar{v}\left(t_{0}\right) e^{-\lambda\left(t-t_{0}\right)}, \quad t \in\left[t_{m}, t_{m+1}\right) .
$$

Suppose (12) is not true. Then, we define

$$
\bar{t}=\inf \left\{t \in\left[t_{m}, t_{m+1}\right) \mid v(t)>\tilde{M} \bar{v}\left(t_{0}\right) e^{-\lambda\left(t-t_{0}\right)}\right\} .
$$

Since (11) holds for $k=m$, we have

$$
\begin{aligned}
& v\left(t_{m}^{-}\right) \leq \tilde{M} \bar{v}\left(t_{0}\right) e^{-\lambda\left(t_{m}-t_{0}\right)}, \\
& v\left(t_{m}^{-}+s\right) \leq \tilde{M} \bar{v}\left(t_{0}\right) e^{-\lambda\left(t_{m}+s-t_{0}\right)}, \quad s \in[-\tau, 0) .
\end{aligned}
$$

Hence

$$
\bar{v}\left(t_{m}^{-}\right) \leq \tilde{M} \bar{v}\left(t_{0}\right) e^{-\lambda\left(t_{m}-\tau-t_{0}\right)} .
$$

From (6) and (13), we have

$$
\begin{aligned}
v\left(t_{m}\right) & \leq\left(a_{m}+b_{m} e^{\lambda \tau}\right) \tilde{M} \bar{v}\left(t_{0}\right) e^{-\lambda\left(t_{m}-t_{0}\right)} \\
& \leq d_{m} \tilde{M} \bar{v}\left(t_{0}\right) e^{-\lambda\left(t_{m}-t_{0}\right)} \\
& =d_{m} \tilde{M} \bar{v}\left(t_{0}\right) e^{\lambda\left(\bar{t}-t_{m}\right)} e^{-\lambda\left(\bar{t}-t_{0}\right)} \\
& <d_{m} \tilde{M} \bar{v}\left(t_{0}\right) e^{\lambda\left(t_{m+1}-t_{m}\right)} e^{-\lambda\left(\bar{t}-t_{0}\right)} \\
& <\tilde{M} \bar{v}\left(t_{0}\right) e^{-\lambda\left(\bar{t}-t_{0}\right)}
\end{aligned}
$$

where the last inequality comes from (ii). Hence, $\bar{t}>t_{m}$, and by the continuity of $v(t)$ in the interval $\left[t_{m}, t_{m+1}\right)$ we have

$$
v(\bar{t})=\tilde{M} \bar{v}\left(t_{0}\right) e^{-\lambda\left(\bar{t}-t_{0}\right)}, \quad v(t) \leq v(\bar{t}), \quad t \in\left[t_{m}, \bar{t}\right] .
$$

From (14), there exists some $t^{*} \in\left(t_{m}, \bar{t}\right)$ such that

$$
\begin{aligned}
& v\left(t^{*}\right)=d_{m} \tilde{M} \bar{v}\left(t_{0}\right) e^{\lambda\left(t_{m+1}-t_{m}\right)} e^{-\lambda\left(\bar{t}-t_{0}\right)}, \\
& v\left(t^{*}\right) \leq v(t) \leq v(\bar{t}), \quad t \in\left[t^{*}, \bar{t}\right] .
\end{aligned}
$$

On the other hand, for any $t \in\left[t^{*}, \bar{t}\right], s \in[-\tau, 0]$, either $t+s \in\left[t_{0}-\tau, t_{m}\right)$ or $t+s \in\left[t_{m}, \bar{t}\right]$.

If $t+s \in\left[t_{0}-\tau, t_{m}\right)$, we obtain

$$
\begin{aligned}
v(t+s) & \leq \tilde{M} \bar{v}\left(t_{0}\right) e^{-\lambda\left(t+s-t_{0}\right)} \\
& \leq \tilde{M} \bar{v}\left(t_{0}\right) e^{-\lambda\left(\bar{t}-t_{0}\right)} e^{\lambda(\bar{t}-t)} e^{\lambda \tau} \\
& \leq e^{\lambda\left(t_{m+1}-t_{m}\right)} \tilde{M} \bar{v}\left(t_{0}\right) e^{-\lambda\left(\bar{t}-t_{0}\right)} e^{\lambda \tau} .
\end{aligned}
$$

If $t+s \in\left[t_{m}, \bar{t}\right]$ from [15], we obtain

$$
\begin{aligned}
v(t+s) & \leq \tilde{M} \bar{v}\left(t_{0}\right) e^{-\lambda\left(\bar{t}-t_{0}\right)} \\
& \leq e^{\lambda\left(t_{m+1}-t_{m}\right)} \tilde{M} \bar{v}\left(t_{0}\right) e^{-\lambda\left(\bar{t}-t_{0}\right)} e^{\lambda \tau} .
\end{aligned}
$$

Hence, from the condition (ii), for any $s \in[-\tau, 0]$ we have

$$
\begin{aligned}
v(t+s) & \leq v\left(t^{*}\right) e^{\lambda \tau} / d_{m} \\
& \leq v(t) e^{\lambda \tau} / d_{m}, \quad t \in\left[t^{*}, \bar{t}\right] .
\end{aligned}
$$

Finally, by the condition (i) as well as $(6)$ and $(16)$, we have

$$
\begin{aligned}
D^{+} v(t) & \leq\left(\alpha+\beta e^{\lambda \tau} / d_{m}\right) v(t) \\
& \leq(\sigma-\lambda) v(t), \quad t \in\left[t^{*}, \bar{t}\right] .
\end{aligned}
$$

According to (ii), we have

$$
\begin{aligned}
v(\bar{t}) \leq & v\left(t^{*}\right) e^{(\sigma-\lambda)\left(\bar{t}-t^{*}\right)} \\
= & d_{m} \tilde{M} \bar{v}\left(t_{0}\right) e^{\lambda\left(t_{m+1}-t_{m}\right)} e^{-\lambda\left(\bar{t}-t_{0}\right)} e^{(\sigma-\lambda)\left(\bar{t}-t^{*}\right)} \\
< & e^{-(\sigma+\lambda)\left(t_{m+1}-t_{m}\right)} \tilde{M} \bar{v}\left(t_{0}\right) e^{\lambda\left(t_{m+1}-t_{m}\right)} \\
& \times e^{-\lambda\left(\bar{t}-t_{0}\right)} e^{(\sigma-\lambda)\left(\bar{t}-t^{*}\right)} \\
= & e^{-\sigma\left(t_{m+1}-t_{m}\right)} \tilde{M} \bar{v}\left(t_{0} e^{-\lambda\left(\bar{t}-t_{0}\right)} e^{(\sigma-\lambda)\left(\bar{t}-t^{*}\right)}\right. \\
< & \tilde{M} \bar{v}\left(t_{0}\right) e^{-\lambda\left(\bar{t}-t_{0}\right)}=v(\bar{t})
\end{aligned}
$$

which is a contradiction. Hence, (12) holds. By induction, we can obtain that 111 holds for any $k \in \mathbb{N}$. This completes the proof.

Remark 1. The impulsive differential inequality was widely used in the stability analysis of dynamic systems including neural networks (Ho et al., 2006; Liu et al., 2005; Xu et al., 2009; Li, 2010; Yang and Xu, 2005; Yang and $\mathrm{Xu}, 2007$; Yue et al., 1999). It is known that $-\alpha>$ $\beta \geq 0$ was assumed by Li (2010), Yang and Xu (2005) or Yue et al. (1999); furthermore, $t_{k}-t_{k-1} \geq \delta \tau$ for some $\delta \geq 1$ was also assumed by Xu et al. (2009), Li (2010) or Yue et al. (1999). However, both of the above-mentioned assumptions are deleted in Lemma 1. The impulsive delay differential inequalities developed by Yang and $\mathrm{Xu}$ (2007) as well as Zhou and Wu (2009) deal with the case without time delay in impulses. Furthermore, to use the method of Yang and Xu (2007), one needs to solve the deduced differential equations, which may be difficult. By Lemma 1, we only need to check the coefficients of the impulsive delay differential inequalities to judge the stability. Hence, from certain perspective, our lemma is less conservative than that of Ho et al. (2006), Liu et al. (2005), Xu et al. (2009), Li (2010), Yang and Xu $(2005 ; 2007)$ or Yue et al. (1999), and extends the famous Halanay differential inequality.

The impulsive differential inequality can be widely used for impulsive control of neural networks and 
stability analysis of impulsive neural networks including Hopfield-type neural networks, BAM neural networks, as well as impulsive control and synchronization of chaotic systems, and so on. In the following, by employing Lemma 1, we shall present several criteria on globally exponential stability for the delayed high-order Hopfield-type neural networks (1) with the impulsive effect. Let $u^{*}=\left[u_{1}^{*}, u_{2}^{*}, \ldots, u_{n}^{*}\right]$ be an equilibrium point of the system (11). Then $x=[0,0, \ldots, 0]^{T}$ is an equilibrium point of system (4) or (5).

Theorem 1. The original point of the high-order Hopfield-type neural networks (5) is globally exponentially stable with the convergence rate $\lambda / 2$ if the following conditions are satisfied:

(i) there exist a matrix $Q>0$, constants $\varepsilon_{1}>0, \varepsilon_{2}>0$ and $\sigma>0, \lambda>0$ such that

$$
\alpha+\left(\beta e^{\lambda \tau}\right) / d_{k-1} \leq \sigma-\lambda
$$

(ii) $\ln d_{k-1}<-(\sigma+\lambda)\left(t_{k}-t_{k-1}\right), k \in \mathbb{N}$, where

$$
\begin{gathered}
\alpha=-\frac{\lambda_{\min }(\Upsilon)}{\lambda_{\max }(Q)}, \\
\beta=\frac{\lambda_{\max }\left(\varepsilon_{1} I+\varepsilon_{2} P_{H}^{T} P_{H}\right) \max _{1 \leq i \leq n}\left\{K_{i}^{2}\right\}}{\lambda_{\min }(Q)}, \\
\Upsilon=-Q C^{-1} R^{-1}-R^{-1} C^{-1} Q+\frac{1}{\varepsilon_{1}} Q C^{-1} P P^{T} C^{-1} Q \\
+\frac{\|M\|^{2}}{\varepsilon_{2}} Q C^{-1} C^{-1} Q,
\end{gathered}
$$

and

$d_{k}$

$$
\begin{aligned}
\geq & 2 \frac{\lambda_{\max }(Q)}{\lambda_{\min }(Q)}\left\|I+D_{k}\right\|^{2} \\
& +2 e^{\lambda \tau} \frac{\lambda_{\max }(Q)}{\lambda_{\min }(Q)} \max _{1 \leq i \leq n}\left\{L_{i}^{2}\right\}\left(\left\|W^{(k)}\right\|+\left\|\Xi_{k}\right\|\|N\|\right)^{2} .
\end{aligned}
$$

Proof. We define a Lyapunov function

$$
v(t)=x^{T}(t) Q x(t) .
$$

Let $t \geq t_{0}$ and $t \in\left[t_{k-1}, t_{k}\right)$. Then for the upper right derivative $D^{+} v$, along the solution of the system (5), we have

$$
\begin{aligned}
D^{+} v(t)= & -x^{T}(t)\left(Q C^{-1} R^{-1}+R^{-1} C^{-1} Q\right) x(t) \\
& +2 x^{T}(t) Q C^{-1} \operatorname{Pf}(x(t-\tau(t))) \\
& +2 x^{T}(t) Q C^{-1} \Gamma^{T} P_{H} f(x(t-\tau(t)))
\end{aligned}
$$

$$
\begin{aligned}
\leq & -x^{T}(t)\left(Q C^{-1} R^{-1}+R^{-1} C^{-1} Q\right) x(t) \\
& +\frac{1}{\varepsilon_{1}} x^{T}(t) Q C^{-1} P P^{T} C^{-1} Q x(t) \\
& +\varepsilon_{1} f^{T}(x(t-\tau(t))) f(x(t-\tau(t))) \\
& +\frac{1}{\varepsilon_{2}} x^{T}(t) Q C^{-1} \Gamma^{T} \Gamma C^{-1} Q x(t) \\
& +\varepsilon_{2} f^{T}(x(t-\tau(t))) P_{H}^{T} P_{H} f(x(t-\tau(t))) .
\end{aligned}
$$

Since $\Gamma^{T} \Gamma=\|\zeta\|^{2} I$ and $\|\zeta\| \leq\|M\|$, from (3), we get

$$
\begin{aligned}
D^{+} v( & t) \\
\leq & -x^{T}(t) \Xi x(t)+f^{T}(x(t-\tau(t))) \\
& \times\left(\varepsilon_{1} I+\varepsilon_{2} P_{H}^{T} P_{H}\right) f(x(t-\tau(t))) \\
\leq & -\lambda_{\min }(\Xi)\|x(t)\|^{2}+\lambda_{\max }\left(\varepsilon_{1} I+\varepsilon_{2} P_{H}^{T} P_{H}\right) \\
& \times \max _{1 \leq i \leq n}\left\{K_{i}^{2}\right\}\|x(t-\tau(t))\|^{2} \\
\leq & -\frac{\lambda_{\min }(\Xi)}{\lambda_{\max }(Q)} v(t) \\
& +\frac{\lambda_{\max }\left(\varepsilon_{1} I+\varepsilon_{2} P_{H}^{T} P_{H}\right) \max _{1 \leq i \leq n}\left\{K_{i}^{2}\right\}}{\lambda_{\min }(Q)} \\
& \times v(t-\tau(t)) \\
\leq & \alpha v(t)+\beta \bar{v}(t) .
\end{aligned}
$$

On the other hand, from the condition (ii), for $k \in \mathbb{N}$,

$$
\begin{aligned}
v( & \left.t_{k}\right) \\
= & \left(x\left(t_{k}^{-}\right)+\Delta x\left(t_{k}^{-}\right)\right)^{T} Q\left(x\left(t_{k}^{-}\right)+\Delta x\left(t_{k}^{-}\right)\right) \\
\leq & \lambda_{\max }(Q) \|\left(I+D_{k}\right) x\left(t_{k}^{-}\right) \\
& +\left(W^{(k)}+\Lambda^{T} \Xi_{k}\right) \varphi\left(x\left(t_{k}^{-}-\tau\left(t_{k}\right)\right)\right) \|^{2} \\
\leq & \lambda_{\max }(Q)\left[\left\|I+D_{k}\right\|\left\|x\left(t_{k}^{-}\right)\right\|\right. \\
& \left.+\left(\left\|W^{(k)}\right\|+\left\|\Lambda^{T}\right\|\left\|\Xi_{k}\right\|\right)\left\|\varphi\left(x\left(t_{k}^{-}-\tau\left(t_{k}\right)\right)\right)\right\|\right]^{2} .
\end{aligned}
$$

By the condition (ii) and the assumptions $\|\varphi(x(t))\| \leq$ $\max _{1 \leq i \leq n}\left\{L_{i}\right\}\|x(t)\|, \Lambda^{T} \Lambda=\|\xi\|^{2} I$ and $\|\xi\| \leq\|N\|$, we obtain

$$
\begin{aligned}
v\left(t_{k}\right) \leq & \lambda_{\max }(Q)\left[\left\|I+D_{k}\right\|\left\|x\left(t_{k}^{-}\right)\right\|\right. \\
& +\max _{1 \leq i \leq n}\left\{L_{i}\right\}\left(\left\|W^{(k)}\right\|\right. \\
& \left.\left.+\left\|\Xi_{k}\right\|\|N\|\right)\left\|x\left(t_{k}^{-}-\tau\left(t_{k}\right)\right)\right\|\right]^{2} \\
\leq & 2 \lambda_{\max }(Q)\left\|I+D_{k}\right\|^{2}\left\|x\left(t_{k}^{-}\right)\right\|^{2} \\
& +2 \lambda_{\max }(Q) \max _{1 \leq i \leq n}\left\{L_{i}^{2}\right\}\left(\left\|W^{(k)}\right\|+\left\|\Xi_{k}\right\|\|N\|\right)^{2} \\
& \times\left\|x\left(t_{k}^{-}-\tau\left(t_{k}\right)\right)\right\|^{2} \\
\leq & 2 \frac{\lambda_{\max }(Q)}{\lambda_{\min }(Q)}\left\|I+D_{k}\right\|^{2} v\left(t_{k}^{-}\right)+2 \frac{\lambda_{\max }(Q)}{\lambda_{\min }(Q)} \\
& \times \max _{1 \leq i \leq n}\left\{L_{i}^{2}\right\}\left(\left\|W^{(k)}\right\|+\left\|\Xi_{k}\right\|\|N\|\right)^{2} \bar{v}\left(t_{k}^{-}\right) .
\end{aligned}
$$

Let

$$
a_{k}=2 \frac{\lambda_{\max }(Q)}{\lambda_{\min }(Q)}\left\|I+D_{k}\right\|^{2},
$$




$$
b_{k}=2 \frac{\lambda_{\max }(Q)}{\lambda_{\min }(Q)} \max _{1 \leq i \leq n}\left\{L_{i}^{2}\right\}\left(\left\|W^{(k)}\right\|+\left\|\Xi_{k}\right\|\|\mid N\|\right)^{2},
$$

$k \in \mathbb{N}$. Then the conditions (i) and (ii) of Lemma1 1 are satisfied from the conditions (i) and (ii). Hence, there exists $\tilde{M}>1$ such that $v(t) \leq \tilde{M} \bar{v}\left(t_{0}\right) e^{-\lambda\left(t-t_{0}\right)}, t \in$ $\left[t_{k-1}, t_{k}\right), k \in \mathbb{N}$.

$$
\|x(t)\| \leq M^{*}|| \phi \|_{\tau} e^{-\lambda\left(t-t_{0}\right) / 2}, t \geq t_{0},
$$

where

$$
M^{*}=\sqrt{\tilde{M} \frac{\lambda_{\max }(Q)}{\lambda_{\min }(Q)}}>1 .
$$

This implies that the equilibrium of the impulsive system (11) is globally exponentially stable and the convergence rate is $\lambda / 2$ for any bounded time delay with $0<\tau<+\infty$. This completes the proof.

Remark 2. Theorem 1 does not require the decreasing of the Lyapunov functions during the continuous portion of the trajectory all the time, which is required by Huang and Yang (2010), and shows that impulses contribute to the exponential stability of some impulsive high-order Hopfield type neural networks with delays. It is also important to emphasize that some existing stability conditions (see, e.g., Ho et al., 2006; Liu and Wang, 20008; Liu et al., 2005; 2007; Khadra et al., 2009; He et al., 2006; Li, 2010; Wang and Liu, 2007) require that every impulsive interval should be bigger than the upper bound of the delay function, while it is indeed dropped here. Thus, the proposed results are more practically applicable since they can be applied to deal with globally exponential stability of neural networks for any time-varying delay with $\tau \in(0,+\infty)$. Moreover, in the works of Liu et al. (2005), $\mathrm{Li}$ (2010), Yang and $\mathrm{Xu}$ (2005) as well as Yue et al. (1999), $\alpha+\beta<0$ is required to be satisfied. According to the condition (i) in Theorem 11 $\alpha+\beta$ can be positive, zero or negative, which shows less conservativeness of our results from certain perspective.

\section{Numerical examples}

The obtained stability conditions contain all the information of neural networks, and only depend on physical parameters of neural networks, which can be checked easily and quickly. Since the new deduced stability criteria can remove some complex restrictions on the relations between impulse times and delays, they are applicable in many practically important problems, such as the analysis of the dynamics of biological neural systems with time-varying delays and the design of globally exponentially stable artificial neural networks.

In the following, we will give two examples to illustrate the advantages of our results.
Example 1. Consider the high-order Hopfield-type neural networks with time-varying delays (1) with $n=$ 3 in much the same way as in the example of (Liu et al., 2005); $t_{k}-t_{k-1}=0.1, k \in \mathbb{N}$ and $0 \leq \tau_{i}(t) \leq$ 0.2 . The parameters of the neural networks are given as follows:

$$
\begin{aligned}
& g_{1}\left(u_{1}\right)=\tanh \left(0.06 u_{1}\right), \\
& g_{2}\left(u_{2}\right)=\tanh \left(0.05 u_{2}\right) \text {, } \\
& g_{3}\left(u_{3}\right)=\tanh \left(0.06 u_{3}\right) \text {, } \\
& h_{1}\left(u_{1}\right)=\tanh \left(0.09 u_{1}\right) \text {, } \\
& h_{2}\left(u_{2}\right)=\tanh \left(0.02 u_{2}\right) \text {, } \\
& h_{3}\left(u_{3}\right)=\tanh \left(0.17 u_{3}\right) \text {, } \\
& C=\operatorname{diag}\left(C_{1}, C_{2}, C_{3}\right)=I \text {, } \\
& R=\operatorname{diag}\left(R_{1}, R_{2}, R_{3}\right)=I \text {, } \\
& I_{1}=I_{2}=0 \text {. } \\
& D_{k}=\operatorname{diag}\left(d_{1 k}, d_{2 k}, d_{3 k}\right) \\
& =\operatorname{diag}(-0.95,-0.84,-0.99) \text {, } \\
& P=\left[\begin{array}{ccc}
0.98 & 1.81 & 6.69 \\
1.60 & 3.16 & -1.29 \\
0.36 & -1.92 & 2.28
\end{array}\right] \text {, } \\
& P_{1}=\left[\begin{array}{ccc}
0.05 & 0.14 & 0.28 \\
-0.06 & -0.05 & 0.11 \\
-0.24 & -0.06 & -0.09
\end{array}\right] \text {, } \\
& P_{2}=\left[\begin{array}{ccc}
0.29 & -0.10 & -0.35 \\
0.23 & -0.14 & 0.25 \\
0.05 & 0.22 & -0.01
\end{array}\right] \text {, } \\
& P_{3}=\left[\begin{array}{ccc}
-0.23 & 0.07 & 0.03 \\
0.09 & -0.02 & -0.19 \\
0.16 & 0.01 & 0.06
\end{array}\right] \text {, } \\
& W=\left[\begin{array}{ccc}
-0.04 & -0.05 & 0.16 \\
0.19 & -0.17 & -0.02 \\
0.03 & 0.13 & 0.04
\end{array}\right] \text {, } \\
& W_{1}=\left[\begin{array}{ccc}
-0.01 & 0.01 & -0.03 \\
0.08 & -0.09 & 0.07 \\
0.08 & -0.01 & 0.01
\end{array}\right] \text {, } \\
& W_{2}=\left[\begin{array}{ccc}
0.06 & 0 & 0.04 \\
0.04 & -0.07 & 0.07 \\
-0.02 & -0.06 & 0.05
\end{array}\right] \text {, } \\
& W_{3}=\left[\begin{array}{ccc}
0.04 & -0.04 & 0.01 \\
0.02 & 0.05 & -0.05 \\
-0.02 & 0.03 & -0.02
\end{array}\right] .
\end{aligned}
$$

In this case, $M=N=[1,1,1]^{T}, \tau=2, K=$ $\operatorname{diag}(0.06,0.05,0.06), L=\operatorname{diag}(0.09,0.02,0.17)$.

Let $Q=I, \varepsilon_{1}=\varepsilon_{2}=1$ in Theorem 1, by computation, we have $\alpha=0.0063, \beta=0.0067$. Let $\sigma=1.7473, \lambda=1.2977, d_{k}=0.0732$. Then $\alpha+\beta e^{\lambda \tau} / d_{k-1}-(\sigma-\lambda)=-0.8813<0 ; \ln d_{k-1}+$ $(\sigma+\lambda)\left(t_{k}-t_{k-1}\right)=-2.3148<0$. Hence, both (i) and (ii) of Theorem 1 are satisfied. Then, we can conclude 
that the equilibrium point of (1) is globally exponentially stable with the convergence rate 0.6489. Figure 1 shows the trajectories of states of the system with impulsive disturbances, which approach zero with time.

Since the impulsive interval is excessively less than the upper bound of time delays, i.e., $t_{k}-t_{k-1}=0.1<$ $\tau=0.2$, the results of Ho et al. (2006), Liu and Wang (2008), Liu et al. (2005; 2007), Khadra et al. (2009), He et al. (2006), Li (2010) as well as Wang and Liu (2007) cannot be used for stability analysis of Example 1. On the other hand, it should be noticed that $\alpha+\beta=0.013>0$ in Example 1. Hence, the results of Liu et al. (2005), Li (2010), Yang and Xu (2005) as well as Yue et al. (1999) cannot be applied to judge the stability either, since $\alpha+$ $\beta<0$ is assumed therein.
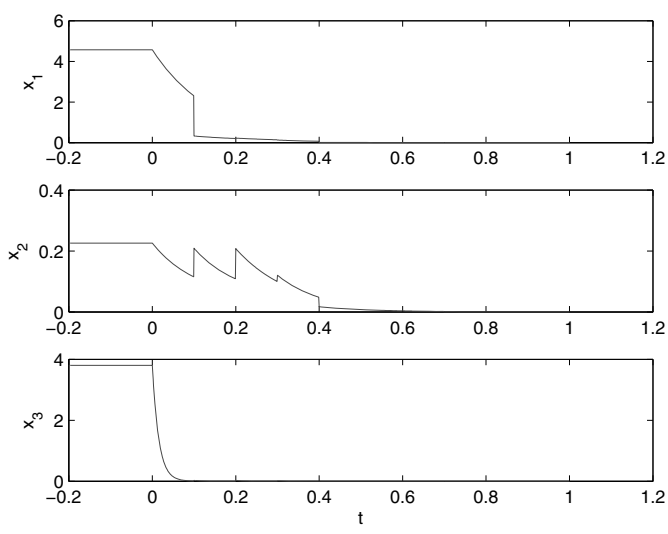

Fig. 1. Time response of states of Example 1 with impulsive disturbances.
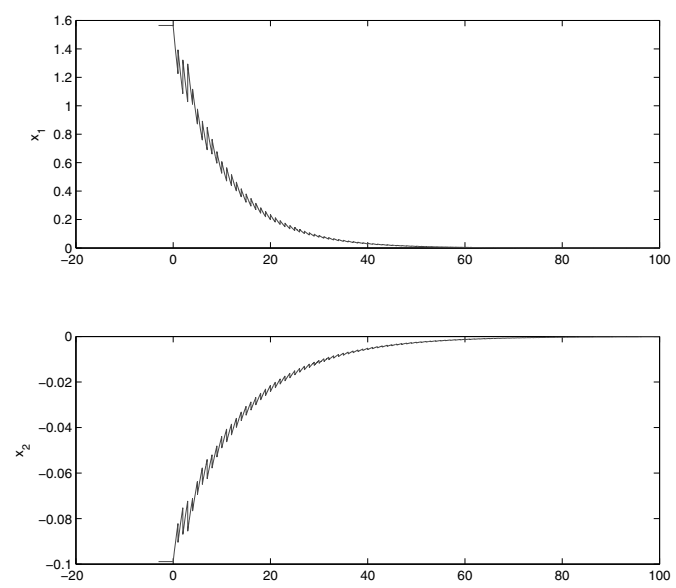

Fig. 2. Time response of states of Example 2 with impulsive disturbances.

Example 2. Consider the high-order Hopfield-type neural networks (11) with $n=2$ with the neuron activation functions and synaptic weights as in the example of Liu and Wang (2008), and $0 \leq \tau_{i}(t) \leq 0.3, t_{k}-t_{k-1}=$ $0.1, k \in \mathbb{N}$. The parameters are given as follows:

$$
\begin{aligned}
g_{1}\left(u_{1}\right) & =\tanh \left(0.1 u_{1}\right), \\
g_{2}\left(u_{2}\right) & =\tanh \left(0.1 u_{2}\right), \\
h_{1}\left(u_{1}\right) & =-\left(u_{1}+u_{2}\right) / 2, \\
h_{2}\left(u_{2}\right) & =\left(u_{1}-u_{2}\right) / 2, \\
R^{-1} & =\operatorname{diag}(0.2,0.1852), \\
D_{k} & =\operatorname{diag}\left(d_{1 k}, d_{2 k}\right)=\operatorname{diag}(-0.5,-0.5), \\
P & =\left(P_{i j}\right)=\left[\begin{array}{cc}
-1 & 0.6 \\
0.9 & -0.9
\end{array}\right], \\
W & =\left(W_{i j}\right)=\left[\begin{array}{cc}
-0.5 & 0.5 \\
-0.5 & -0.5
\end{array}\right], \\
C_{1} & =C_{2}=1, \quad P_{1}=P_{2}=0, \\
W_{1} & =W_{2}=0, \quad I_{1}=I_{2}=0 .
\end{aligned}
$$

In this case, $M=[1,1]^{T}, N=[1.6,0.6]^{T} \tau=$ $2, K=\operatorname{diag}(0.1,0.1), L=0$.

Let $Q=0.2079 I, \varepsilon_{1}=1.2506, \varepsilon_{2}=1.0438$. Вy computation, we have $a=0.1852, \mu=0.0213$, [ $[\mid I+$ $\left.D_{k} \|+\max _{1 \leq i \leq n}\left\{L_{i}\right\}\left(\left\|W^{(k)}\right\|+\left\|\Xi_{k}\right\|\|N\|\right)\right]^{2}=0.25$. Let $\sigma=0.6, \lambda=0.2, d_{k}=0.5$. Then $\alpha+\beta e^{\lambda \tau} / d_{k-1}-$ $(\sigma-\lambda)=-0.3263<0, \ln d_{k-1}+(\sigma+\lambda)\left(t_{k}-t_{k-1}\right)=$ $-0.6131<0$. Then, we see that the equilibrium point of (11) is globally exponentially stable with the convergence rate 0.1 by Theorem 1 Figure 2 shows the stability of states of the system with impulsive disturbances.

The stability conclusion for this example cannot be derived by applying the corresponding results of Liu and Wang (2008), Xu et al. (2009; 2011) Huang and Yang (2010), Zhang et al. (2010) or Li (2010), since the impulses considered here are nonlinear and the delay in the impulses is time-varying. Since the impulsive interval is excessively less than the upper bound of time delays, i.e., $t_{k}-t_{k-1}=0.1<\tau=0.3$, the results of Ho et al. (2006), Liu and Wang (2008), Liu et al. (2005; 2007), Khadra et al. (2009), He et al. (2006), Li (2010) or Wang and Liu (2007) cannot be used for the stability analysis of this example, either. Furthermore, $\alpha+\beta=0.0513>0$ here, then, as Example 1, the results of Liu et al. (2005), $\mathrm{Li}$ (2010), Yang and Xu (2005) as well as Yue et al. (1999) are useless to judge the stability.

Remark 3. In biological neural networks, impulsive effects are likely to exist. For example, when stimulus from the body or the external environment is received by receptors, the electrical impulses will be conveyed to the neural net and impulsive effects arise naturally in the net. Therefore, a neural network model with delays and impulsive effects should be more accurate to describe the evolutionary process of the systems. Then the impulsive differential inequality obtained in the paper can be applied to analyse the stability of neural networks. 


\section{Conclusion}

A new impulsive differential inequality has been derived and sufficient conditions for globally exponential stability of the equilibrium point for delayed high-order Hopfield-type neural networks with impulsive effects have been presented. The proposed results have been proved theoretically and experimentally to be less conservative than some existing stability criteria for impulsive neural networks.

Furthermore, since genes play a leading role in the control of cellular processes, gene regulatory networks have become a hot research area in the biological and biomedical sciences and have received great attention. It would be interesting to study the stability of genetic regulatory networks with impulses. As one of powerful methods to analyze the stability, the obtained impulsive differential inequality will be used to investigate the stability of genetic regulatory networks in our future research.

\section{Acknowledgment}

The authors wish to thank the editor and the reviewers for a number of constructive comments and suggestions that have improved the quality of the paper. This work was supported the NNSF of China (Grants No. 61175119, 11101373, 11271333 and 61074011), the Program for New Century Excellent Talents in University (NCET-10-0329), the Alexander von Humboldt Foundation of Germany, the NSF of Jiangsu Province, China (Grant No. BK2010408), and the Zhejiang Innovation Project, China (Grant No. T200905).

\section{References}

Ahmad, S. and Stamova, I. (2008). Global exponential stability for impulsive cellular neural networks with time-varying delays, Nonlinear Analysis: Theory, Methods \& Applications 69(3): 786-795.

Cao, J. and Xiao, M. (2007). Stability and Hopf bifurcation in a simplified BAM neural network with two time delays, IEEE Transactions on Neural Networks 18(2): 416-430.

Chen, T. (2001). Global exponential stability of delayed Hopfield neural networks, Neural Networks 14(8): 977-980.

Chua, L. and Yang, L. (1988a). Cellular neural networks: Applications, IEEE Transactions on Circuits and Systems 35(10): 1273-1290.

Chua, L. and Yang, L. (1988b). Cellular neural networks: Theory, IEEE Transactions on Circuits and Systems 35(10): 1257-1272.

Civalleri, P., Gilli, M. and Pandolfi, L. (1993). On stability of cellular neural networks with delay, IEEE Transactions on Circuits and Systems I: Fundamental Theory and Applications 40(3): 157-165.
Gopalsamy, K. and He, X. (1994). Stability in asymmetric Hopfield nets with transmission delays, Physica D: Nonlinear Phenomena 76(4): 344-358.

Halanay, A. (1966). Differential Equations: Stability, Oscillations, Time Lags, Academic Press, New York, NY.

He, Y., Wang, Q., Wu, M. and Lin, C. (2006). Delay-dependent state estimation for delayed neural networks, IEEE Transactions on Neural Networks 17(4): 1077-1081.

Ho, D., Liang, J. and Lam, J. (2006). Global exponential stability of impulsive high-order BAM neural networks with time-varying delays, Neural Networks 19(10): 1581-1590.

Huang, Z. and Yang, Q. (2010). Exponential stability of impulsive high-order cellular neural networks with time-varying delays, Nonlinear Analysis: Real World Applications 11(1): 592-600.

Khadra, A., Liu, X. and Shen, X. (2009). Analyzing the robustness of impulsive synchronization coupled by linear delayed impulses, IEEE Transactions on Automatic Control 54(4): 923-928.

Li, C., Feng, G. and Huang, T. (2008). On hybrid impulsive and switching neural networks, IEEE Transactions on Systems, Man, and Cybernetics, Part B: Cybernetics 38(6): 1549-1560.

Li, X. (2010). Global exponential stability of delay neural networks with impulsive perturbations, Advances in Dynamical Systems and Applications 5(1): 107-122.

Liu, X., Shen, X., Zhang, Y. and Wang, Q. (2007). Stability criteria for impulsive systems with time delay and unstable system matrices, IEEE Transactions on Circuits and Systems I: Regular Papers 54(10): 2288-2298.

Liu, X., Teo, K. and Xu, B. (2005). Exponential stability of impulsive high-order Hopfield-type neural networks with time-varying delays, IEEE Transactions on Neural Networks 16(6): 1329-1339.

Liu, X. and Wang, Q. (2008). Impulsive stabilization of high-order Hopfield-type neural networks with time-varying delays, IEEE Transactions on Neural Networks 19(1): 71-79.

Liu, Y., Zhao, S. and Lu, J. (2011). A new fuzzy impulsive control of chaotic systems based on T-S fuzzy model, IEEE Transactions on Fuzzy Systems 19(2): 393-398.

Lou, X. and Cui, B. (2007). Novel global stability criteria for high-order Hopfield-type neural networks with time-varying delays, Journal of Mathematical Analysis and Applications 330(1): 144-158.

Lu, J., Ho, D. and Cao, J. (2010). A unified synchronization criterion for impulsive dynamical networks, Automatica 46(7): 1215-1221.

Lu, J., Ho, D., Cao, J. and Kurths, J. (2011). Exponential synchronization of linearly coupled neural networks with impulsive disturbances, IEEE Transactions on Neural Networks 22(2): 329-336.

Lu, J., Kurths, J., Cao, J., Mahdavi, N. and Huang, C. (2012). Synchronization control for nonlinear stochastic dynamical networks: Pinning impulsive strategy, IEEE 
Transactions on Neural Networks and Learning Systems 23(2): 285-292.

Raja, R., Sakthivel, R., Anthoni, S.M. and Kim, H. (2011). Stability of impulsive Hopfield neural networks with Markovian switching and time-varying delays, International Journal of Applied Mathematics and Computer Science 21(1): 127-135, DOI: 10.2478/v10006-011-0009-y.

Ren, F. and Cao, J. (2006). LMI-based criteria for stability of high-order neural networks with time-varying delay, Nonlinear Analysis: Real World Applications 7(5): 967-979.

Rong, L. (2005). LMI approach for global periodicity of neural networks with time-varying delays, IEEE Transactions on Circuits and Systems I: Regular Papers 52(7): 1451-1458.

Sakthivel, R., Raja, R. and Anthoni, S. (2011). Exponential stability for delayed stochastic bidirectional associative memory neural networks with markovian jumping and impulses, Journal of Optimization Theory and Applications 150(1): 166-187.

Sakthivel, R., Samidurai, R. and Anthoni, S. (2010a). Asymptotic stability of stochastic delayed recurrent neural networks with impulsive effects, Journal of Optimization Theory and Applications 147(3): 583-596.

Sakthivel, R., Samidurai, R. and Anthoni, S. (2010b). Exponential stability for stochastic neural networks of neutral type with impulsive effects, Modern Physics Letters B 24(11): 1099-1110.

Sakthivel, R., Raja, R. and Anthoni, S. (2010c). Asymptotic stability of delayed stochastic genetic regulatory networks with impulses, Physica Scripta 82(5): 055009.

Stamova, I. and Ilarionov, R. (2010). On global exponential stability for impulsive cellular neural networks with time-varying delays, Computers \& Mathematics with Applications 59(11): 3508-3515.

Stamova, I., Ilarionov, R. and Vaneva, R. (2010). Impulsive control for a class of neural networks with bounded and unbounded delays, Applied Mathematics and Computation 216(1): 285-290.

Tian, Y., Yu, X. and Chua, L. (2004). Time-delayed impulsive control of chaotic hybrid systems, International Journal of Bifurcation and Chaos in Applied Sciences and Engineering 14(3): 1091-1104.

van den Driessche, P. and Zou, X. (1998). Global attractivity in delayed Hopfield neural network models, SIAM Journal on Applied Mathematics 58(6): 1878-1890.

Wallis, G. (2005). Stability criteria for unsupervised temporal association networks, IEEE Transactions on Neural Networks 16(2): 301-311.

Wang, Q. and Liu, X. (2007). Exponential stability of impulsive cellular neural networks with time delay via Lyapunov functionals, Applied Mathematics and Computation 194(1): 186-198.

Weng, A. and Sun, J. (2009). Impulsive stabilization of second-order nonlinear delay differential systems, Applied Mathematics and Computation 214(1): 95-101.
Wu, B., Liu, Y. and Lu, J. (2011). Impulsive control of chaotic systems and its applications in synchronization, Chinese Physics B 20(5): 050508.

Wu, B., Liu, Y. and Lu, J. (2012a). New results on global exponential stability for impulsive cellular neural networks with any bounded time-varying delays, Mathematical and Computer Modelling 55(3-4): 837-843.

Wu, B., Han, J. and Cai, X. (2012b). On the practical stability of impulsive differential equations with infinite delay in terms of two measures, Abstract and Applied Analysis 2012: 434137.

Xu, B., Liu, X. and Liao, X. (2003). Global asymptotic stability of high-order hopfield type neural networks with time delays, Computers \& Mathematics with Applications 45(10-11): 1729-1737.

Xu, B., Liu, X. and Teo, K. (2009). Asymptotic stability of impulsive high-order hopfield type neural networks, Computers \& Mathematics with Applications 57(11-12): 1968-1977.

Xu, B., Xu, Y. and He, L. (2011). LMI-based stability analysis of impulsive high-order Hopfield-type neural networks, Mathematics and Computers in Simulation, DOI: 10.1016/j.matcom.2011.02.008.

Yang, T. and Chua, L. (1997). Impulsive stabilization for control and synchronization of chaotic systems: Theory and application to secure communication, IEEE Transactions on Circuits and Systems I: Fundamental Theory and Applications 44(10): 976-988.

Yang, Z. and Xu, D. (2005). Stability analysis of delay neural networks with impulsive effects, IEEE Transactions on Circuits and Systems II: Express Briefs 52(8): 517-521.

Yang, Z. and Xu, D. (2007). Stability analysis and design of impulsive control systems with time delay, IEEE Transactions on Automatic Control 52(8): 1448-1454.

Yue, D., Xu, S. and Liu, Y. (1999). Differential inequality with delay and impulse and its applications to design robust control, Journal of Control Theory and Applications 16(4): 519-524.

Zhang, H., Ma, T., Huang, G. and Wang, Z. (2010). Robust global exponential synchronization of uncertain chaotic delayed neural networks via dual-stage impulsive control, IEEE Transactions on Systems, Man, and Cybernetics, Part B: Cybernetics 40(3): 831-844.

Zhang, Q., Yang, L. and Liao, D. (2011). Existence and exponential stability of a periodic solution for fuzzy cellular neural networks with time-varying delays, International Journal of Applied Mathematics and Computer Science 21(4): 649-658, DOI: 10.2478/v10006-011-0051-9.

Zhang, Y. and Sun, J. (2010). Stability of impulsive linear hybrid systems with time delay, Journal of Systems Science and Complexity 23(4): 738-747.

Zheng, C., Zhang, H. and Wang, Z. (2011). Novel exponential stability criteria of high-order neural networks with time-varying delays, IEEE Transactions on Systems, Man and Cybernetics, Part B: Cybernetics 41(2): 486-496. 
Zhou, J. and Wu, Q. (2009). Exponential stability of impulsive delayed linear differential equations, IEEE Transactions on Circuits and Systems II: Express Briefs 56(9): 744-748.

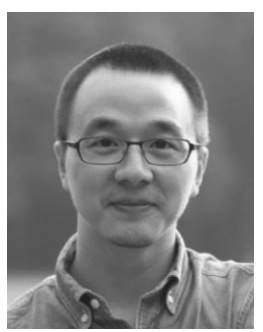

Yang Liu received the B.Sc. degree in mathematics from Zhejiang Normal University, China, in 2003, and the Ph.D. degree from Tongji University, Shanghai, in 2008. He is currently an associate professor at the Department of Mathematics, Zhejiang Normal University. His research interests cover complex analysis, Clifford analysis, switched systems, delayed systems and nonlinear control. He has published over 20 papers in refereed international journals and is a recipient of the 2011 Shanghai Outstanding Ph.D. Thesis Award.

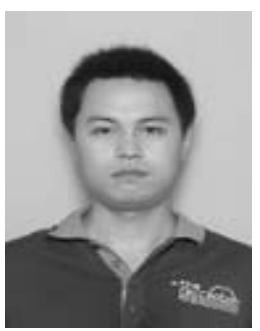

Rongjiang Yang is a master student at the Department of Mathematics, Zhejiang Normal University. His research interest covers nonlinear control and its applications.

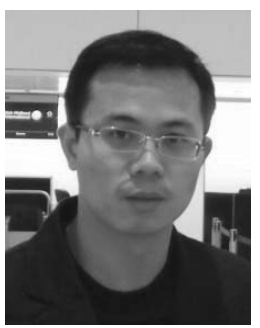

Jianquan Lu received the B.Sc. degree in mathematics from Zhejiang Normal University, China, in 2003, the M.Sc. degree in mathematics from Southeast University, Nanjing, China, in 2006, and the Ph.D. degree from the City University of Hong Kong, Hong Kong, in 2009. He is currently an associate professor at the Department of Mathematics, Southeast University. His present research interests include collective behavior in complex dynamical networks, multiagent systems, and nonlinear systems and control. He has published over 30 papers in refereed international journals. Dr. Lu is an associate editor of Neural Processing Letters and Neural Computing and Applications, and a guest editor of Mathematics and Computers in Simulation. $\mathrm{He}$ is a recipient of the Jiangsu Provincial Outstanding Master's Thesis Award (2007), a TOP 100 Most Cited Chinese Papers Published in International Journals distinction (2007), an Alexander von Humboldt Fellowship (2010), the Program for New Century Excellent Talents in University by the Ministry of Education, China (2010), and the First Award of Jiangsu Provincial Progress in Science and Technology (2010).

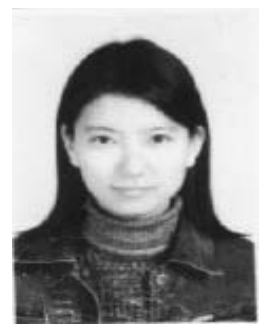

Bo Wu received the B.Sc. and the M.Sc. degrees in mathematics from Zhejiang Normal University, China, in 2003 and 2006, respectively. She is currently a lecturer at the Academic Affairs Division, Zhejiang Normal University. Her research interest covers neural networks and nonlinear control.

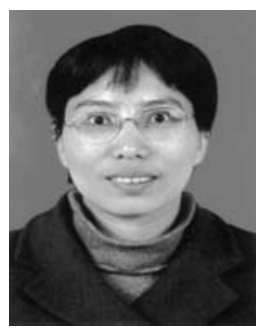

Xiushan Cai received her B.Sc. degree in mathematics from Fujiang Normal University, China, in 1988, an M.Sc. degree in operation science and control theory from East China Normal University, China, in 1999 and a Ph.D degree in control theory and control engineering from Shanghai Jiao Tong University, China, in 2005. She is currently a professor of Zhejiang Normal University, China. Her research interests include nonlinear systems theory, optimisation, robust control theory and control applications in mechanical systems.

Received: 31 December 2011 Revised: 9 May 2012 